\title{
A educação em saúde no pré-natal: conhecimento das gestantes sobre as posições maternas durante o parto normal
}

\author{
Prenatal health education: knowledge of pregnant women about maternal positions during \\ normal birth
}

\section{Educación en salud prenatal: conocimiento de las mujeres embarazadas sobre las posiciones maternas durante el parto normal}

Mariana Moura dos Santos Gomes ${ }^{1 *}$, Fernando Kleber Martins Barbosa ${ }^{2}$, Camilla Cristina Lisboa do Nascimento ${ }^{2}$, Yury Gomes ${ }^{2}$, Karoline Nobre de Lima ${ }^{1}$, Vanessa Santos Ferreira ${ }^{1}$, Lucas Ferreira de Oliveira, Márcia Simão Carneiro', Elisângela da Silva Ferreira ${ }^{1}$, Ana Paula Oliveira Gonçalves.

\section{RESUMO}

Objetivo: Identificar e analisar o conhecimento de gestantes sobre as posições de parir no processo de parturição, antes e após uma ação educativa no pré-natal. Apresentar as características sócio econômicas das participantes do estudo, identificar se o conhecimento das gestantes sobre as posições de parir estão de acordo com as Diretrizes do Parto e Nascimento do Ministério da Saúde e avaliar o aprendizado destas gestantes após ação educativa. Métodos: Estudo qualitativo, utilizando-se a metodologia da pesquisa-ação. Foram aplicados dois questionários de perguntas abertas e fechadas, após isso foi realizado a ação educativa com objetivo de fornecer informações sobre a temática proposta. Resultados: Emergiram duas categorias denominadas Vantagens do parto normal na concepção das gestantes e Conhecimento das gestantes sobre o trabalho de parto e as posições para parir. Esta pesquisa poderá trazer benefícios para a comunidade acadêmica, aos serviços de saúde e enfermeiros, políticas públicas e para as gestantes pela possiblidade de propagação do conhecimento sobre o assunto. Conclusão: Este estudo possibilitou compreender a importância da educação em saúde no pré-natal, assim como o papel do profissional enfermeiro nesse contexto. Foi possível observar mudanças e ensinamentos através da metodologia empregada em um curto prazo, através das respostas positivas das participantes.

Palavras-Chave: Educação em saúde, Cuidado pré-natal, Parto.

\section{ABSTRACT}

Objective: To identify and analyze the knowledge of pregnant women about the positions of giving birth in the parturition process, before and after an educational action in prenatal care. To present the socio-economic characteristics of the study participants, identify whether the knowledge of pregnant women about the positions of giving birth are in accordance with the Birth and Birth Guidelines of the Ministry of Health and evaluate the learning of these pregnant women after educational action. Methods: Qualitative study, using the action research methodology. Two questionnaires with open and closed questions were applied, after which an educational action was carried out to provide information on the proposed theme. Results: Two categories emerged called Advantages of normal childbirth in the conception of pregnant women and Knowledge of pregnant women about labor and the positions to give birth. This research can bring benefits to the academic community, health services and nurses, public policies and to pregnant women due to the possibility of spreading knowledge on the subject. Conclusion: This study made it possible to understand the importance of health education in prenatal care, as well as the role of the professional nurse in this context. It was possible to observe changes and teachings through the methodology used in a short term, through the positive responses of the participants.

Key words: Health education, Prenatal care, Childbirth.

${ }^{1}$ Universidade Federal do Pará (UFPA), Belém - PA. *E-mail: marianamouraenf@gmail.com

2 Universidade do Estado do Pará (UEPA), Belém - PA. 


\section{RESUMEN}

Objetivo: Identificar y analizar el conocimiento de las mujeres embarazadas sobre las posiciones de dar a luz en el proceso de parto, antes y después de una acción educativa en la atención prenatal. Para presentar las características socioeconómicas de los participantes del estudio, identifique si el conocimiento de las mujeres embarazadas acerca de las posiciones de dar a luz está de acuerdo con las Pautas de nacimiento y parto del Ministerio de Salud y evalúe el aprendizaje de estas mujeres embarazadas después de la acción educativa. Métodos: Estudio cualitativo, utilizando la metodología de investigación de acción. Se aplicaron dos cuestionarios con preguntas abiertas y cerradas, después de lo cual se llevó a cabo una acción educativa para proporcionar información sobre el tema propuesto. Resultados: Surgieron dos categorías llamadas Ventajas del parto normal en la concepción de mujeres embarazadas y Conocimiento de las mujeres embarazadas sobre el trabajo de parto y las posiciones para dar a luz. Esta investigación puede aportar beneficios a la comunidad académica, los servicios de salud y las enfermeras, las políticas públicas y las mujeres embarazadas debido a la posibilidad de difundir conocimientos sobre el tema. Conclusión: Este estudio permitió comprender la importancia de la educación para la salud en la atención prenatal, así como el papel de la enfermera profesional en este contexto. Fue posible observar cambios y enseñanzas a través de la metodología utilizada a corto plazo, a través de las respuestas positivas de los participantes.

Palabras clave: Educación para la salud, Atención prenatal, Parto.

\section{INTRODUÇÃO}

O objetivo do acompanhamento pré-natal segundo Ministério da Saúde (2012) é garantir o desenvolvimento da gestação, permitindo o parto de um bebê saudável, sem impacto para a saúde materna, inclusive realizando um acompanhamento que aborde aspectos psicossociais e as atividades educativas e preventivas. Ainda, segundo Ministério da Saúde (2012) no pré-natal é realizado a avaliação do estado da gestante por meio da verificação de peso, altura, pressão arterial, sendo anotado os dados no cartão da gestante, fornecimento de medicações conforme prescrição médica ou do enfermeiro, solicitação de exames laboratoriais, coleta de exame preventivo, aplicação de vacina, medição da altura uterina, avaliação de movimentos fetais e ausculta dos batimentos cardio-fetais.

Para Nunes JT, et al., (2016) o pré-natal é um fator fundamental na prevenção e proteção de eventos adversos sobre a saúde obstétrica, onde pode permitir a identificação e o manuseio clínico de intervenções oportunas sobre potenciais riscos para complicações na saúde da mãe e do bebê, através de consultas durante os trimestres da gestação. Carneiro MS (2012), descreve em seu estudo que o enfermeiro tem um papel fundamental na assistência ao parto e durante o pré-natal, nos cuidados oferecidos a mãe e bebê, possuindo a oportunidade de levar a promoção da saúde e prevenção de morbidades. A autora relaciona o pré-natal com o agir cuidativo e educativo do enfermeiro. Ainda de acordo com o autor supracitado, dentre os temas educativos que podem ser abordados, estes devem abranger as necessidades da gestante, as técnicas não farmacológicas para o alívio da dor e boas práticas humanizadas no trabalho de parto. Dentre as boas práticas, as posições que podem optar no momento do parto para benefício do processo de nascimento.

É importante considerarmos também a explanação do contexto histórico e social de parto e nascimento. Nos estudos de Souza SACB (2014), o parto e nascimento são considerados marco histórico na sociedade por ser evento natural que modifica e marca a vida de mulheres e suas famílias. Para esse autor, o marco histórico é caracterizado por atitudes e comportamentos aprendidos na sociedade que revelam a cultura enraizada de um povo. Nesse contexto de influências históricas, Souza SACB (2014) afirma que é possível observar a intensa medicalização do parto e a perda da autonomia da mulher, que resulta na mudança no papel de protagonista para coadjuvante do parto inerente ao modelo obstétrico institucionalizado. $O$ autor caracteriza esse modelo quando a mulher colabora com os procedimentos definidos e realizados pelos profissionais que a assistem, os comportamentos adotados são definidos, assim como a maneira e a posição de parir. O momento ideal para garantir a aprendizagem da gestante e família sobre o cuidado do seu corpo e do bebê, assim como compreender o processo do trabalho de parto e parto é durante o pré-natal. 
Cortês CT, et al., (2015) afirma que os profissionais devem orientar durante todo o pré-natal, garantindo a mulher $o$ acesso a essas informações, devendo escolher e assumir a posição que desejar durante o trabalho de parto e parto. As evidências científicas demonstram através de pesquisas qualitativas e quantitativas que a fisiologia do parto natural e em posições verticalizadas, trazem mais benefícios e vantagens na hora do momento de parir. Conforme Araújo BM (2013) o parto vaginal pode ser em várias posições, não somente na tradicional posição ginecológica ou de decúbito dorsal. Nesse aspecto a parturiente pode experimentar várias posições para parir, pode ficar de quatro apoios, pode experimentar ficar em decúbito lateral esquerdo com pernas flexionadas, escolher ficar verticalizada, em posição semi-verticalizada e de cócoras. Cortês CT, et al., (2015), a importância de estimular as posições verticalizadas contribuem para a garantia de partos mais fisiológicos, que reduz o desconforto e a dificuldade de puxos, tornando o parto menos dolorido e reduzindo o traumatismo vaginal ou perineal no período expulsivo.

Silveira IP e Carvalho FAM (2003) afirmam que as posições de parir na vertical ajudam a pelve a atingir maior abertura, favorecem ao aumento das contrações uterinas, favorece a ação da força gravitacional, aumentam os puxos que facilitam na descida e expulsão do bebê, diminui a distensão do períneo posterior, diminuiu a incidência de episiotomias e de roturas uterinas. Além de favorecerem o relaxamento da mulher, inibindo a limitação do sacro, facilitando assim o movimento de báscula. É necessário que as gestantes possuam conhecimentos sobre o parto normal, assistências e condutas adequadas, posições em que elas podem escolher parir para assim fortalecer seu protagonismo em seus direitos e escolhas.

Diante disso o objetivo desse estudo é analisar as vantagens do parto normal na concepção das gestantes e o conhecimento das gestantes sobre as posições de parir antes e após uma ação educativa no pré-natal.

\section{MÉTODOS}

Trata-se de um estudo de natureza qualitativa, utilizando-se a metodologia da pesquisa- ação, prática que associa pesquisadores e atores em uma mesma estratégia de ação para modificar uma dada situação e uma estratégia de pesquisa para adquirir um conhecimento sistemático sobre a situação identificada (FRANCO MAS, 2005). As participantes do estudo foram 16 gestantes que são atendidas no programa de pré-natal na Unidade Municipal de Saúde da Cremação, escolhidas por amostra aleatória e saturação das informações.

Os dados foram coletados por meio de dois questionários com perguntas fechadas e abertas, elaborados pela pesquisadora. No primeiro questionário que foi aplicado antes da ação educativa os conteúdos das perguntas fechadas abordaram os seguintes aspectos: dados pessoais (naturalidade, idade, escolaridade, estado civil, religião), dados sociais (renda familiar, situação conjugal atual, se possui filhos e quantos), se a mulher já possui filhos, qual foi ou foram as vias de parto.

Ainda sobre as perguntas fechadas outros aspectos mencionados foram: se existem vantagens no parto normal para a mulher e criança, se durante o pré- natal a mulher recebeu alguma informação sobre o trabalho de parto e se sim, marcar quais foram, se ela possuía desejo de optar pela via de parto normal e selecionar quais posições para parir ela conhece (quatro apoios, cócoras, joelhos, sentada, semi-sentada, lateralizada, litotomia e em pé) e se ela gostaria de receber informações sobre as variedades de posições.

Através da escrita das gestantes, foi possível identificar o conhecimento adquirido dessas mulheres na vivência do pré-natal sobre as posições no processo de parir. Podemos assim ter um comparativo entre o instrumento de avaliação antes da ação educativa, e o mesmo instrumento após a ação educativa, sendo as discussões baseadas segundo as Diretrizes Nacionais de Assistência ao Parto Normal de 2017.

A pesquisa atendeu aos preceitos éticos da Resolução n. 466/2012 do Conselho Nacional de Saúde. Foi aprovada no Comitê de ética e Pesquisa do Instituto de Ciências da Saúde da Universidade Federal do Pará (UFPA) sob o parecer de número 3.567.847 e CAAE número 14898819.1.0000.0018. Foi submetido para autorização pela Secretaria Municipal de Saúde (SESMA), após as suas respectivas aprovações se deu início ao estudo. Foi apresentado para cada puérpera uma cópia do Termo de Consentimento Livre e Esclarecido (TCLE), para ser assinado, e foi assegurado o anonimato das entrevistadas por meio de pseudônimos que as identificaram durante toda a pesquisa. 


\section{RESULTADOS E DISCUSSÃO}

O maior quantitativo de participantes do estudo encontrava-se na faixa etária de 16 a 32 anos, sendo que a média aritmética do total de idade foi de 25,5 anos, com a maior parte $(43,75 \%)$ entre 27 a 32 anos ( 7 gestantes). Em relação a escolaridade, o estudo evidenciou que a maioria das gestantes possuíam um significativo grau de instrução, cerca de $43,75 \%$ (7 gestantes), possuíam o ensino médio completo, onde esse dado é seguido do segundo maior percentual de $25 \%$ (4 gestantes) que possuíam o ensino superior completo.

Quanto ao estado civil a maioria eram solteiras (62,5\% - 10 gestantes); $25 \%$ eram casadas (4 gestantes) e 12,5\% (2 gestantes) estavam em união estável. Tendo como renda familiar de $43,75 \%$ (7) dessas gestantes era de 1 a 2 salários mínimos (Tabela 1).

Tabela 1 - Características socioeconômicas das participantes do estudo. Belém - Pará, 2020.

\begin{tabular}{|c|c|c|}
\hline \\
\hline & TOTAL & TOTAL \\
\hline Características & $\mathbf{N}=16$ & $\%=100$ \\
\hline \multicolumn{3}{|l|}{ Faixa etária } \\
\hline $16-20$ anos & 3 & $18,75 \%$ \\
\hline 23-26 anos & 6 & $37,5 \%$ \\
\hline 27-32 anos & 7 & $43,75 \%$ \\
\hline \multicolumn{3}{|l|}{ Escolaridade } \\
\hline Analfabeto & 0 & $0 \%$ \\
\hline Ensino fundamental incompleto & 2 & $12,5 \%$ \\
\hline Ensino médio incompleto & 1 & $6,25 \%$ \\
\hline Ensino médio completo & 7 & $43,75 \%$ \\
\hline Ensino superior incompleto & 2 & $12,5 \%$ \\
\hline Ensino superior completo & 4 & $25 \%$ \\
\hline \multicolumn{3}{|l|}{ Religião } \\
\hline Católica & 6 & $37,5 \%$ \\
\hline Evangélica & 7 & $43,75 \%$ \\
\hline Sem religião & 2 & $12,5 \%$ \\
\hline Não informou & 1 & $6,25 \%$ \\
\hline \multicolumn{3}{|l|}{ Estado Civil } \\
\hline Solteira & 10 & $62,5 \%$ \\
\hline Casada & 4 & $25 \%$ \\
\hline União estável & 2 & $12,5 \%$ \\
\hline Viúva & 0 & $0 \%$ \\
\hline Separada & 0 & $0 \%$ \\
\hline \multicolumn{3}{|l|}{ Renda familiar } \\
\hline Nenhuma & 0 & $0 \%$ \\
\hline Até 1 salário mínimo & 6 & $37,5 \%$ \\
\hline 1 - 2 salários & 7 & $43,75 \%$ \\
\hline 2 - 5 salários & 2 & $12,5 \%$ \\
\hline Mais de 5 salários & 0 & $0 \%$ \\
\hline Não soube especificar & 1 & $6,25 \%$ \\
\hline
\end{tabular}

Fonte: Gomes MMS, et al., 2020. 
No que se refere ao número de filhos, 56,25\% (9 gestantes) possuem de 1 a 2 filhos e $43,75 \%$ ( 7 gestantes) ainda não possuem filhos nascidos. Destas que possuem filhos nascidos, 93,75\% (7 gestantes) tiveram seus filhos de parto normal e 22,5\% (2 gestantes) de cesariana. O estudo ainda evidenciou que cerca de $77,77 \%$ (7 gestantes) tiveram parto normal e 22,22 \% (9 gestantes) cesariana (Tabela 2).

Tabela 2 - Quantitativo de filhos e tipos de parto das participantes do estudo. Belém - Pará, 2020.

\section{TOTAL TOTAL}

\section{Características}

\begin{tabular}{lcc} 
& $\mathbf{N}=\mathbf{1 6}$ & $\%=\mathbf{1 0 0}$ \\
\hline Número de filhos & & 43,75 \\
\hline Nenhum filho & 7 & 56,25 \\
1-2 filhos & 9 & 0 \\
3- 4 filhos & 0 & 0 \\
$5-6$ filhos & 0 & 0 \\
Mais de 6 filhos & 0 & $\%=100$ \\
\hline Tipos de parto & $\mathbf{N}=\mathbf{9}$ & $\mathbf{7 7 , 7}$ \\
\hline Normal & 7 & 22,2 \\
Cesariana & 2 & 0 \\
Natural & 0 & 0 \\
Na água & 0 & 0 \\
Fórceps & 0 &
\end{tabular}

Fonte: Gomes MMS, et al., 2020.

Ao realizar a análise as categorias emergiram a partir das perguntas formuladas no instrumento de coleta de dados e consequente respostas, que serão apresentadas posteriormente, os dados obtidos revelaram em seus resultados aspectos que demonstraram vantagens do parto normal na concepção das gestantes e o conhecimento das gestantes sobre as posições de parir correlacionando-as com a facilidade de expulsão do bebê e lacerações na vagina. No primeiro momento, antes da ação educativa obteve-se os seguintes resultados em relação as categorias.

\section{Categoria 1 - Vantagens do parto normal na concepção das gestantes}

Das 16 participantes do estudo, quando indagadas se existem vantagens do parto normal para a mulher e crianças, 15 responderam que sim e 1 respondeu que não. Essa última não informou o motivo de não achar vantagens no parto normal. Ao serem perguntadas quais seriam as vantagens do parto normal, a maior significação foi a recuperação mais rápida no pós-parto. Essa percepção pode ser ilustrada nas falas a seguir:

"A recuperação no pós-parto é mais rápida para a mãe e logo após o nascimento se pode ter imediatamente o contato com o bebê." (E1, 27 anos).

"Melhor e mais rápida recuperação, contato mais próximo com o bebê." (E2, 28 anos).

"A vantagem do parto normal é a mulher ter uma recuperação mais rápida do que uma cesariana." (E3, 20 anos)."

Esse achado vai ao encontro com outras pesquisas que comprovam a visão das mulheres sobre as vantagens do parto normal. Para os autores Santana FA, Lahm JV e Santos RP (2015) entre as mulheres entrevistadas, algumas consideravam vantagem no parto normal pela recuperação mais rápida, devido deixar a mulher mais independente nos cuidados com o bebê. 
Na pesquisa de Kottwits F, et al. (2018) esse fato torna-se ainda mais expressivo, mediante seu estudo realizado com 361 puérperas que desejaram optar pelo parto normal, dentre os motivos da preferência, 229 puérperas desejaram o parto normal devido a recuperação mais rápida no pós-parto.

Dentro da significação da recuperação mais rápida no pós-parto, percebemos por meio das respostas que a maioria também atrelou essa vantagem ao contato e vínculo afetivo maior com o recém-nascido nos primeiros momentos após o parto. Nesse sentido, entende-se que para as gestantes pesquisadas, o fato de atrelarem a recuperação mais rápida no pós-parto normal com o contato e vínculo com o bebê, demonstra 0 extinto materno de criar esse vínculo afetivo logo no momento do nascimento e a necessidade do contato pele a pele na primeira hora de vida, para ambos se sentirem em conexão afetiva.

Sobre o vínculo afetivo e contato, os autores Sampaio ARR, Bosquat A e Barros C (2016), citam que o contato pele a pele precoce entre mãe e bebê apresenta benefícios comprovados a curto e longo prazo para mãe e para a criança Cunha ACB, et al. (2012), afirmam que o vínculo entre mãe e bebê proporciona a criança experimentar sentimentos de confiança, segurança e bem-estar, sendo fundamental para o desenvolvimento infantil. Nessa linha racional, os autores Fucks IS, et al. (2015) referem que cabe toda a equipe de saúde desde o pré-natal até o processo de parturição, assegurar o momento de vínculo afetivo e que seja vivenciado na sua integralidade para que traga benefícios para mãe, bebê e a família

Ainda sobre as vantagens do parto normal, dentre as entrevistadas, uma delas apontou um outro significado, conforme descrito a seguir:

\section{"Respeitar o tempo natural do bebê e a recuperação pós-parto." (E12,32 anos).}

Essa afirmativa da participante nos chama a atenção na pesquisa por possivelmente ela ter um conhecimento teórico prévio sobre o assunto ou por ter um conhecimento empírico no quesito do momento em que o bebê estará pronto para nascer e dará os primeiros sinais de chegada. O momento que o bebê dará sinais de manifestação de seu nascimento, podem ser traduzidos pelos sinais e sintomas de trabalho de parto no corpo da gestante, para Félix HCR, et al. (2019) o reconhecimento do verdadeiro trabalho consiste na presença de contrações uterinas rítmicas e em intervalos regulares que não 45 cessam e aumentam gradativamente com perda do tampão mucoso e concomitante dilatação do colo uterino.

\section{Categoria 2 - Conhecimento das gestantes sobre o trabalho de parto e as posições para parir}

Essa categoria trata do conhecimento das gestantes sobre o trabalho de parto e as posições para parir. A temática das perguntas que provocaram a essa categoria foram: qual o conhecimento sobre o trabalho de parto e as posições para parir; qual a relação entre a posição de parir com a facilidade de expulsão do bebê sem causar lacerações na vagina e se elas obtiveram orientações sobre o trabalho de parto e parto no prénatal.

Mediante as respostas, evidenciou-se o conhecimento das gestantes que realizam o pré-natal possuíam sobre o trabalho de parto e as posições para parir no parto normal até aquele momento antes da intervenção educativa. As respostas de um modo geral provavelmente revelam o senso comum sobre o assunto, devido possivelmente apresentar reflexos da qualidade do processo de educação em saúde realizado durante o prénatal.

"Quando está sangrando tem que ir pro hospital; tem que deitar e fazer força pra baixo [...]". (E4, 25 anos).

"Eu só pensava que existia deitada." (E5, 31 anos).

"[...] a posição da mulher sempre de perna aberta fazendo esforço de expulsar se preciso." (E6, 24 anos).

A prevalência da posição litotômica e conhecimento de práticas desatualizadas como o puxo dirigido foram evidenciadas na maioria das falas das entrevistadas, essas falas corroboram o pensamento de Almeida NAM, et al. (2012), que referem a utilização de práticas obstétricas medicalizadas e institucionalizadas na obstetrícia brasileira. 
As novas evidências cientificas e atualização das boas práticas do parto e nascimento segundo as Diretrizes Nacionais (2017), devem fazer parte da rotina dos profissionais que atuam no cenário obstétrico, para fornecer subsídios e orientação a todos os envolvidos no cuidado, no intuito de promover, proteger e incentivar o parto normal. O repasse de orientações e informações atuais para gestantes e puérperas devem ser realizadas por profissionais atualizados, ainda no pré-natal.

Sobre as informações acerca do processo do trabalho de parto, se teve como resultado quando indagadas, se receberam informações sobre o processo do trabalho de parto durante a realização do pré-natal, das 16 entrevistadas, 8 relataram que receberam orientações; 7 responderam que não receberam orientações e uma entrevistada não lembra. Esse quantitativo de mulheres que receberam informações e o tipo de informações recebidas pode ser mais bem visualizado por meio da tabela abaixo (Tabela 3 ).

Tabela 3 - Informações sobre o processo do trabalho de parto e as posições para parir com as 16 entrevistadas da pesquisa. Belém - Pará, 2020.

\begin{tabular}{cccc} 
Receberam informações & SIM & NÃO & NÃO LEMBRA \\
& 8 & 7 & 0 \\
\hline
\end{tabular}

\section{Tipos de informações}

\begin{tabular}{llll}
\hline Sinais e sintomas de trabalho de parto. & 6 & - & - \\
$\begin{array}{l}\text { Duração do trabalho de parto em média, de } \\
\text { acordo com o número de partos anteriores. }\end{array}$ & 1 & - & - \\
$\begin{array}{l}\text { Perdas vaginais e dilatação do colo do útero } \\
\text { até o nascimento do bebê. }\end{array}$ & 3 & - & - \\
Posições de parir. & 4 & - & - \\
Direito a acompanhante. & 4 & - & - \\
Violência Obstétrica. & 1 & - & - \\
Intervenções que podem ocorrer durante um & 1 & - & - \\
parto difícil. & & & - \\
Aleitamento na primeira hora pós-parto. & 4 &
\end{tabular}

Fonte: Gomes MMS, et al., 2020.

Dentre as que receberam orientações a maioria foram sobre sinais e sintomas do trabalho de parto, em seguida de posições de parir, direito a acompanhante e aleitamento na primeira hora pós-parto. O segundo momento foi após a roda de conversa com as gestantes, o qual a intervenção educativa foi a explanação da temática oralmente e através de slides em Power Point abordando a variedade de posições para parir e os benefícios de cada posição para mãe e para o bebê. Onde foram elencadas as mesmas categorias do primeiro momento antes da ação educativa para realizar os comparativos do antes e depois da intervenção educativa.

\section{Categoria 1 - Vantagens do parto normal na concepção das gestantes}

Essa categoria emergiu a partir das respostas das entrevistadas através do segundo instrumento de coleta de dados após a ação educativa. Observamos um acréscimo teórico na opinião sobre o assunto após a ação educativa. No primeiro momento essa categoria abordou a significação da recuperação pós-parto, porém nesse segundo momento as participantes já trouxeram uma nova significação como vantagem para o parto normal, que estão evidenciadas nas falas abaixo:

"Que o melhor parto é o normal, pois tenho como escolher algumas posições para parir, que facilita o bebê nascer." (E8, 25 anos).

"Sobre o parto normal é que é mais benéfico, tanto para a mãe quanto para o bebê e que as posições verticalizadas são mais eficazes na hora do parto." (E14, 29 anos). 
Para Silva DF, et al., (2018) a mulher deve ter liberdade da escolha da posição que melhor lhe convém, favorecendo todos os aspectos do trabalho de parto e quando bem indicadas não serão negativas para o estado geral do recém-nascido. Essa vantagem detectada pelas participantes após a roda de conversa foi concernente a Ministério da Saúde (2017), cuja Diretrizes do Parto e Nascimento incentivam as mulheres a adotarem qualquer posição, incluindo as posições verticalizadas.

\section{Categoria 2 - Conhecimento das gestantes sobre o trabalho de parto e as posições para parir}

Nessa categoria quando indagadas novamente sobre o que conhecem sobre as posições de parir, emergiram as seguintes respostas:

"Existem diversas posições entre deitada e em pé, porém a gestante decide qual é a mais confortável, e é provado que as verticais são as mais indicadas por facilitar o parto" (E10, 27 anos).

"Deve sempre beneficiar a mulher, posições que se sinta confortável e que facilite a saída do bebê" (E7, 24 anos).

"Agora com a informação dada eu sei que tem variantes de posições de parir, não é só deitada. E que não somos obrigadas a parir em uma só posição" (E6, 31 anos).

Após a ação educativa é possível salientar o embasamento teórico que vão ao encontro das Diretrizes do Parto e Nascimento (2017) que incentivam as posições verticalizadas e a escolha da posição de parir que se sentir mais confortável. Além disso, Silva DF, et al., (2018) afirma que a posição vertical auxilia o alívio da dor durante o trabalho de parto, melhora o conforto e satisfação da mulher durante o parto, tendo a mulher um maior controle sobre o seu corpo. Os autores supramencionados salientam que cabe aos profissionais orientar quanto as posições mais eficazes e confortáveis.

Grande parte dos sujeitos da pesquisa escolheriam a posição em pé, de cócoras e quatro apoios no seu trabalho de parto e período expulsivo. Essas escolhas das participantes vão de encontro com o estímulo das posições verticalizadas das (DIRETRIZES NACIONAIS DO PARTO E NASCIMENTO, 2017). Na posição de cócoras segundo Silva (2014) há maior diâmetro da pelve materna além de facilitar na descida e rotação do bebê, fortalecendo o assoalho pélvico, aumentando a oxigenação fetal e fortalecendo a musculatura perineal.

Segundo os resultados obtidos na pesquisa de Souza VB, et al. (2011) a comunicação, informação e troca de saberes entre profissionais e gestantes devem ser priorizadas na assistência pré-natal em todas as circunstâncias, devido serem a forma de promover a compreensão de todo o ciclo gestacional. Para Jardim MJA (2017) a educação em saúde através do profissional enfermeiro tem a capacidade de sensibilizar a gestante, influenciando positivamente o desfecho obstétrico dessa mulher, mudando a concepção e mentalidade sobre a maternidade e o processo do parto.

Araújo BM (2013) menciona sobre a atuação do enfermeiro na assistência de enfermagem no pré-natal, o qual possui papel de executar medidas e condutas preventivas, educativas e curativas, objetivando a gestante e familiares bem-estar físico, psíquico e social, além do acompanhamento das características biológicas. Diante disso a educação em saúde através do profissional enfermeiro tem a capacidade de sensibilizar a gestante, influenciando positivamente o desfecho obstétrico dessa mulher, mudando a concepção e mentalidade sobre a maternidade e o processo do parto.

\section{CONCLUSÃO}

Este estudo nos possibilitou compreender a importância da educação em saúde no pré-natal, assim como o papel da equipe multiprofissional do pré-natal e do profissional enfermeiro, adotando a educação em saúde como rotina em suas práticas, com objetivo de repassar conhecimentos as gestantes, e atender todas as suas necessidades no seu ciclo gravídico e puerperal. Ao avaliarmos o aprendizado das gestantes após a ação educativa por meio das rodas de conversas e da aplicação do instrumento idêntico ao aplicado antes da ação educativa, foi possível observar mudanças obtendo respostas positivas. Espera-se que esse estudo possa contribuir com boas práticas de educação em saúde no pré natal e possibilitar pesquisas voltadas ao tema. 


\section{REFERÊNCIAS}

1. ALMEIDA NAM, et al. Perspectivas de dor do parto normal de primigestas no período pré natal. Texto Contexto Enfermagem, 2012; 21(4): 819-27.

2. ARAÚJO B.M. Educação para o parto: o enfermeiro no preparo das mulheres durante o pré-natal.2013. Trabalho de Conclusão de Curso (Bacharelado em Enfermagem) - Universidade Federal Fluminense, 2013; 70.

3. CARNEIRO MS. Representações sociais sobre pré-natal entre mulheres-mães do Pará: implicações para o agir cuidativo-educativo em enfermagem. Dissertação (Mestrado em educação e tecnologia de enfermagem para o cuidado em saúde a indivíduos e grupos sociais) - Universidade do Estado Pará (UEPA) e Universidade Federal do Amazonas (UFAM), Belém, 2012; p67.

4. CORTÊS CT. et. al. Metodologia de implementação de práticas baseadas em evidências científicas na assistência ao parto normal: um estudo piloto. Revista da escola de enfermagem USP, 2015; 49(5): 716- 725.

5. CUNHA ACB, SANTOS C e GONÇALVES RM. Concepções sobre maternidade, parto e amamentação em grupo de gestantes. Arquivos Brasileiros de Psicologia, Rio de Janeiro v.64, n.1, p. 139-155, 2012.

6. FÉLIX HCR, et al. Sinais de alerta e de trabalho de parto: conhecimento entre gestantes. Revista Brasileira de Saúde Materno Infantil, Recife, v. 19, n.2, p. 343- 349 abr-jun., 2019.

7. FRANCO MAS. Pedagogia da pesquisa- ação. Educação e Pesquisa.2005; 31(3): 483-502.

8. FUCKS IS, et al. A sala de parto: o contato pele a pele e as ações para o estímulo ao vínculo entre mãe-bebê. Av Enferm. v.33, n.1, p. 29-37, 2015.

9. JARDIM MJA, et al. Contribuições do enfermeiro para o empoderamento da gestante no processo de parturição natural. VIII Jornada Internacional Políticas Públicas, UFMA. 2017.

10. KOTTWITZ F, et al. Via de parto preferida por puérperas e suas motivações. Revista Escola Anna Nery, 2018; 22(1): 2017-0013.

11. MINISTÉRIO DA SAÚDE. Cadernos de Atenção Básica. Atenção ao pré-natal de baixo risco. Brasília-DF; 2012. 65p.

12. Ministério da Saúde. Diretrizes Nacionais de Assistência ao Parto Normal. Brasília, 2017; 87p.

13. NUNES JT, et al. Qualidade da assistência pré-natal no Brasil: revisão de artigos publicados de 2005 a 2015 . Caderno Saúde Coletiva, 2016; 24(123): 252-61.

14. SAMPAIO ARR, BOSQUAT A e BARROS C. Contato pele a pele ao nascer: um desafio para a promoção do aleitamento materno em maternidade pública no Nordeste brasileiro com o título de Hospital Amigo da Criança. Epidemiol. Serv. Saude, Brasília, v.25, n.2, p.281-290, abr-jun 2016.

15. SANTANA FA, et al. Fatores que influenciam a gestante na escolha do tipo de parto. Rev. Fac. Ciênc. Méd. Sorocaba,2015; 17(3): $123-127$.

16. SILVA DF, et al. Conhecimento das gestantes sobre as posições do parto. Revista de Enfermagem da FACIPLAC, 2018; 1(1): 130-504.

17. SILVA LS. Os saberes das gestantes acerca das diferentes posições de parir. Trabalho de Conclusão de Curso (Bacharelado e licenciatura em Enfermagem) - Universidade Federal Fluminense, 2014; 89p.

18. SILVEIRA IP e CARVALHO FAM. Conhecimento das mulheres sobre posições para o parto normal. Rev. RENE. 2003; 4(1), 36-39.

19. SOUSA SACB. As implicações das posições maternas no trabalho de parto. Dissertação (Mestrado em Enfermagem de Saúde Materna e Obstetrícia) - Escola Superior de Enfermagem do Porto. Porto, 2014; p78.

20. SOUZA VB, et al. Ações educativas durante a assistência pré-natal: percepção de gestantes atendidas na rede básica de Maringá-PR. Revista Eletrônica de Enfermagem, 2011; 13(2): 199-219. 